\title{
Manejo diagnóstico y terapéutico del insulinoma pancreático
}

\author{
P. Priego, A. Sanjuanbenito, E. Martínez Molina, E. Lobo, D. García Teruel, V. Morales, G. Rodríguez y \\ V. Fresneda \\ Departamento de Cirugía General y Digestivo. Hospital Ramón y Cajal. Madrid
}

\section{RESUMEN}

Introducción: el insulinoma es el tumor endocrino pancreático más frecuente. Su diagnóstico preoperatorio ha sido motivo de controversia durante muchos años. El objetivo del trabajo es evaluar la experiencia del Hospital Ramón y Cajal en el manejo diagnóstico y terapéutico de este tipo de tumores.

Material y métodos: en el periodo comprendido entre enero de 1999 y julio de 2006 fueron intervenidos en nuestro hospital 12 pacientes ( 9 mujeres y 3 varones) con el diagnóstico de insulinoma, con una edad media de 56 años (16-72 años).

Resultados: la gammagrafía con octreótido nos permitió realizar el diagnóstico en el 33,3\% de los casos, el TAC abdominal en el $83,3 \%$ y la ecoendoscopia en el 100\%.

El $100 \%$ de los insulinomas fue diagnosticado durante la cirugía gracias al uso de la ecografía intraoperatoria. En todos los casos se realizó la excisión completa del tumor, mediante 9 enucleaciones y 3 pancreatectomías distales.

Dos pacientes presentaron una fístula pancreática y otro un pseudoquiste pancreático que se resolvieron de forma conservadora. La tasa de curación fue del 100\%. El seguimiento fue de 48 meses, no encontrándose ningún caso de recidiva.

Conclusión: en nuestra experiencia, creemos aconsejable la realización de TAC abdominal y ecoendoscopia como pruebas previas a la cirugía.

Sin embargo, la técnica de elección sería la palpación del páncreas y la realización de una ecografía intraoperatoria.

La cirugía es curativa en el $100 \%$ de los casos, y la técnica efectuada dependerá del tamaño, localización y distancia del tumor al conducto pancreático, así como su relación con el síndrome MEN-1.

Palabras clave: Insulinoma. Tumor endocrino pancreático. Ecografía intraoperatoria. Ecoendoscopia.

\begin{abstract}
Introduction: insulinoma is the most frequent pancreatic endocrine tumor. Its preoperative diagnosis has been controversial for many years. The aim of this study was to evaluate the experience in the management and treatment of this kind of tumor at Hospital Ramón y Cajal.

Material and methods: between January 1999 and July 2006, 12 patients were operated on in our hospital (9 females and 3 males) who had been diagnosed with insulinoma, with a mean age of 56 years (16-72 years).

Results: octreotide scintigraphy allowed a diagnosis in 33.3\% of cases, abdominal CT in $83.3 \%$, and echoendoscopy in $100 \%$. Intraoperative ultrasonography confirmed the presence of an insulinoma in $100 \%$ of cases. In all cases a complete excision was possible, with 9 enucleations and 3 distal pancreatectomies.

Two patients developed a pancreatic fistula, and one a pancreatic pseudocyst that healed spontaneously without surgery. The overall cure rate was $100 \%$. After a mean follow-up of 48 months no recurrences have been reported.

Conclusion: in our experience, we consider advisable that abdominal CT and echoendoscopy be performed before surgery. However, the initial procedure of choice would be pancreas palpation and intraoperative ultrasonography. Surgery cured 100\% of cases, and the procedure selected depends on size, location, distance from the main pancreatic duct, and relation to multiple endocrine neoplasm 1 (MEN-1).
\end{abstract}

Key words: Insulinoma. Pancreatic endocrine tumor. Intraoperative ultrasonography. Echoendoscopy.
Recibido: 26-09-06.

Aceptado: 21-12-06.

Correspondencia: Pablo Priego Jiménez. C/ Fermín Caballero, 26, $1^{\circ} \mathrm{A}$. 16004 Cuenca. e-mail: papriego@hotmail.com
Priego P, Sanjuanbenito A, Martínez Molina E, Lobo E, García Teruel D, Morales, V Rodríguez G, Fresneda V. Manejo diagnóstico y terapéutico del insulinoma pancreático. Rev Esp Enferm Dig 2007; 99: 218-222. 


\section{INTRODUCCIÓN}

El insulinoma es el tumor neuroendocrino funcionante más frecuente del páncreas, siendo su incidencia de 1-2 casos por $10^{6}$ /habitantes al año (1).

La mayor parte de estos tumores son únicos, benignos y de pequeño tamaño.

A pesar del gran número de pruebas de imagen de que disponemos (ecografía, TAC, RMN), el pequeño tamaño de los insulinomas hace que el diagnóstico topográfico no siempre sea posible, presentando en la literatura unas cifras de sensibilidad en el diagnóstico preoperatorio que no sobrepasa el 50-60\%.

La aparición de la ecoendoscopia, junto con la combinación de la palpación digital del páncreas y la ecografía intraoperatoria, han revolucionado el diagnóstico de este tipo de tumores, alcanzando cifras en torno al 90-100\% (2).

El presente estudio tiene como objetivo analizar el manejo diagnóstico y terapéutico del insulinoma en el Hospital Ramón y Cajal en los últimos años.

\section{MATERIAL Y MÉTODOS}

En el periodo comprendido entre enero de 1999 y julio de 2006, fueron intervenidos en el Hospital Ramón y Cajal 12 pacientes con el diagnóstico de insulinoma. De ellos 9 fueron mujeres y 3 varones con edades comprendidas entre los 16-72 años (media de 56 años).

Los pacientes fueron evaluados prospectivamente y se recogieron y analizaron las características clínicas, radiológicas, operatorias y anatomopatológicas.

Todos los pacientes fueron estudiados en el Servicio de Endocrinología de nuestro centro. El diagnóstico de insulinoma se estableció mediante la presencia de síntomas de neurohipoglucemia, hipoglucemia de ayuno, test del ayuno positivo, determinación de la proinsulina y péptido C (Tabla I). También se descartaron otras causas de hiperinsulinismo e hipoglucemia (sulfonilureas, insulina exógena, etc.). No se realizó ningún test de provocación. Los pacientes se mantuvieron euglucémicos hasta la intervención mediante una combinación de dieta, diazóxido en dosis de 200 a $400 \mathrm{mg} /$ día y en dos casos mediante la administración de octreótido a dosis de $200 \mathrm{mg}$ /día.

Entre las pruebas diagnósticas efectuadas en el preoperatorio, realizamos TAC abdominal, gammagrafía con octreótido, ecoendoscopia, RMN y arteriografía mesentérica.

Todos los pacientes fueron intervenidos bajo anestesia general realizándose preferentemente un abordaje mediante una incisión subcostal bilateral.

El páncreas se expuso completamente a través del saco menor y se movilizó de forma que pudiera palparse adecuadamente, cuando el tumor se localizaba en la cabeza pancreática se realizó un kocher amplio.

A continuación se realizó una ecografía intraoperatoria utilizando una sonda de $10 \mathrm{MHz}$, la ecografía fue realiza-

\begin{tabular}{cccccc} 
Tabla I \\
\hline Pacientes & $\begin{array}{c}\text { Glucemia } \\
\text { basal } \\
(\mathrm{mg} / \mathrm{dl})\end{array}$ & $\begin{array}{c}\text { Insulina } \\
\text { plasma } \\
(\mu \mathrm{U} / \mathrm{m} /)\end{array}$ & Péptido C & $\begin{array}{c}\text { Tiempo entre } \\
\text { sintomas- } \\
\text { Diagnóstico }\end{array}$ & $\begin{array}{c}\text { Tiempo entre } \\
\text { sintomas- } \\
\text { Cirugía }\end{array}$ \\
\hline 1 & 36 & 52 & 4,1 & 12 meses & 38 meses \\
2 & 48 & 31,5 & 3,4 & 40 meses & 76 meses \\
3 & 45 & 15 & 2,1 & 3 meses & 5 meses \\
4 & 57 & 26,6 & 4,9 & 40 meses & 66 meses \\
5 & 45 & 42 & 3,12 & 5 meses & 11 meses \\
6 & 38 & 30 & 6,17 & 5 meses & 12 meses \\
7 & 32 & 23 & 1,75 & 36 meses & 44 meses \\
8 & 43 & 44,7 & 2,2 & 12 meses & 24 meses \\
9 & 43 & 55,7 & 12,5 & 30 meses & 50 meses \\
10 & 30 & 35 & 4,69 & 10 meses & 20 meses \\
11 & 35 & 39 & 6,7 & 15 meses & 21 meses \\
12 & 27 & 88 & 5,1 & 8 meses & 14 meses \\
\hline
\end{tabular}

da por el cirujano que contó con la ayuda de un radiólogo en quirófano cuando se creyó necesario. En la ecografía se exploró toda la glándula, prestando atención a la presencia de lesiones multicéntricas así como a la relación del tumor con los vasos esplénicos y mesentéricos y con el conducto de Wirsung. Una vez realizada la ecografía se procedió a la extirpación tumoral, mediante la enucleación de la lesión o mediante una resección pancreática parcial, utilizando tanto en una como en otra técnica, con buenos resultados, el bisturí harmónico. Todos los pacientes pasaron a la UVI quirúrgica durante 24 horas para estricto control de la glucemia.

\section{RESULTADOS}

En 11 de los casos el insulinoma apareció de forma esporádica $(91,6 \%)$ y en uno de los casos asociado a un síndrome MEN-1 $(8,4 \%)$.

En 11 casos se realizó el diagnóstico de insulinoma primario, mientras que otro de los casos se trataba de una recidiva de un insulinoma intervenido en nuestro centro en el año 1998.

En 6 pacientes se realizó gammagrafía con octreótido marcado, siendo positiva en 2 casos.

En el $100 \%$ de los pacientes se realizó un TAC abdominal, que permitió localizar la lesión en 10/12 pacientes $(83,3 \%)$.

La arteriografía mesentérica fue positiva para el diagnóstico del insulinoma en los 2 casos en que se realizó, y lo mismo sucedió en los 3 casos en que se efectuó la ecoendoscopia (Figs. 1 y 2).

La palpación y exploración del páncreas y del tumor, junto con la utilización de la ecografía intraoperatoria consiguió localizar el tumor en el $100 \%$ de los casos, incluso uno de ellos que no había sido localizado preoperatoriamente mediante TAC y RMN (Figs. 3 y 4).

Se realizó enucleación en 9 casos y pancreatectomía distal en 3 casos. En el caso de recidiva, que había sido tratado previamente con enucleación, se realizó resección. 


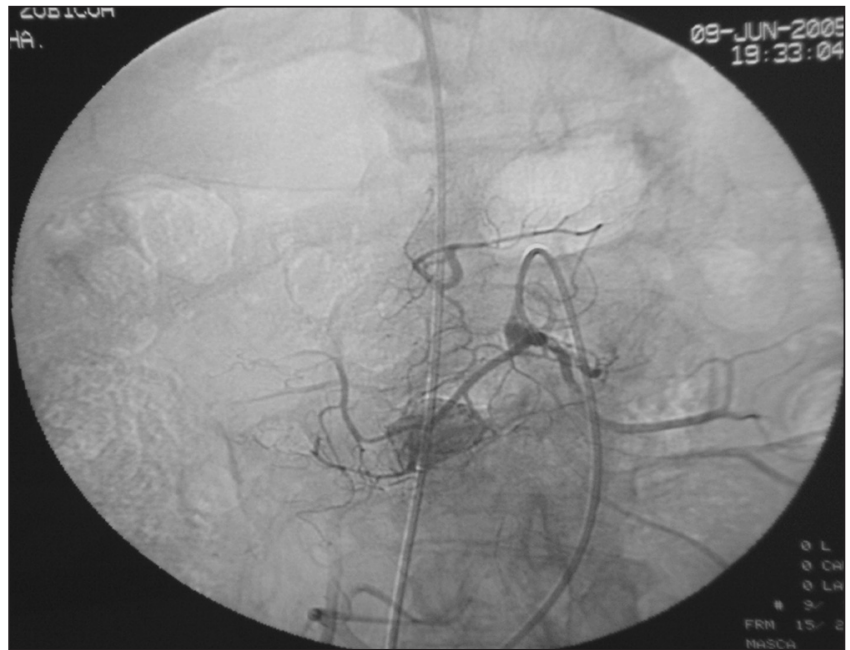

Fig. 1. Arteriografía.

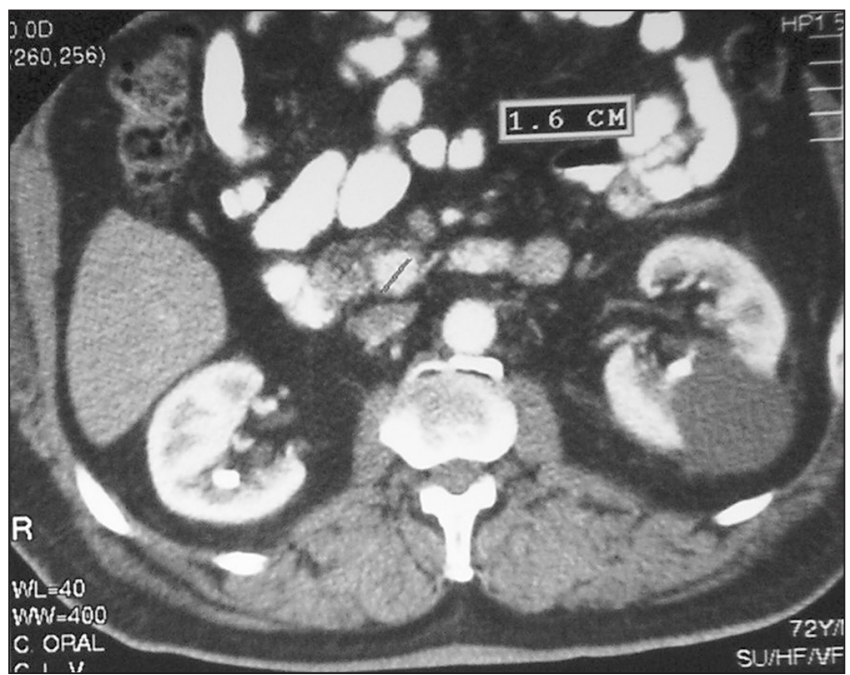

Fig. 2. TAC abdominal.

La duración media del acto quirúrgico fue de $182 \mathrm{mi}-$ nutos (100-270 min), siendo de 179 minutos para la enucleación (100-270 $\min$ ) y de 185 minutos para la resección pancreática (150-230 min).

El estudio anatomopatológico reveló un carácter benigno en el 100\% de las lesiones, y no se encontró ningún caso de lesiones múltiples. El estudio de las imágenes demuestra la siguiente distribución de las lesiones: cabeza (6 casos), cuerpo y cola (6 casos). El tamaño de las lesiones osciló entre 1-2,5 cm con una media de $2 \mathrm{~cm}$.

La tasa de mortalidad fue del $0 \%$. Entre las complicaciones quirúrgicas (3/12: $25 \%$ ), encontramos dos casos de fístula pancreática $(16,6 \%)$, una tras enucleación y otra tras pancreatectomía distal; y otro de pseudoquiste pancreático $(8,3 \%)$ que se resolvieron de forma conservadora.

En todos los pacientes se produjo la desaparición de los síntomas hipoglucémicos, junto con una normaliza-

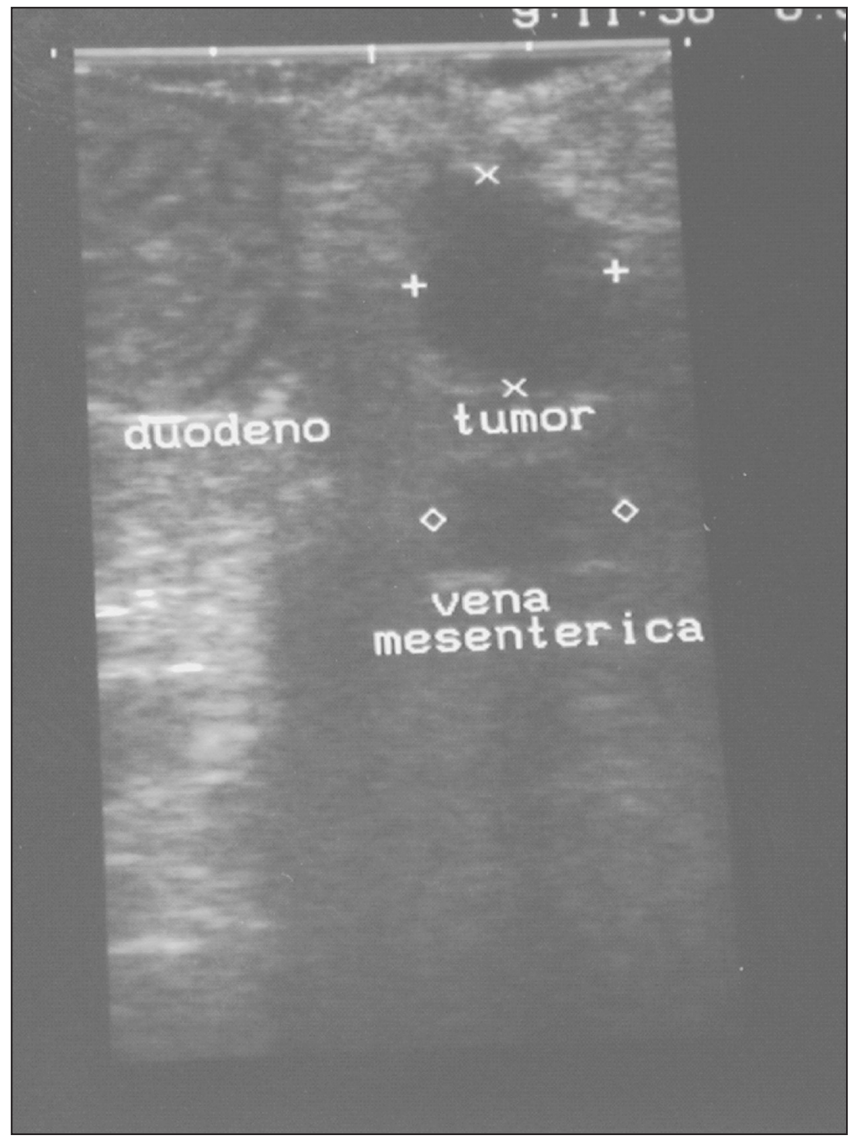

Fig. 3. Ecografía intraoperatoria.

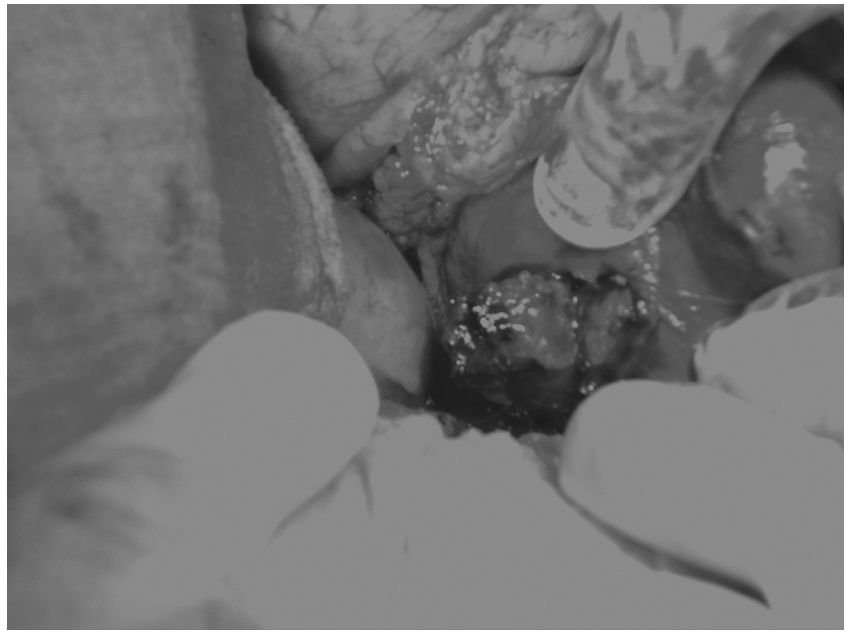

Fig. 4. Insulinoma en cabeza páncreas.

ción de las cifras de glucemia. No se produjeron complicaciones metabólicas en ningún paciente.

El seguimiento medio de estos pacientes fue de 48 meses (1-73 meses), no encontrándose ningún caso de recidiva. 


\section{DISCUSIÓN}

El insulinoma es el tumor endocrino pancreático funcionante más frecuente (1). Su mayor incidencia entre la $3^{\mathrm{a}}-6^{\mathrm{a}}$ década de la vida y su predominio en el sexo femenino se confirman en nuestra serie, en donde la edad media de presentación fue de 56 años y el 75\% fueron mujeres.

Aproximadamente un $90 \%$ son solitarios, siendo en un pequeño porcentaje múltiples, fundamentalmente los relacionados con el síndrome MEN-1 (10\%) (1). Todos nuestros insulinomas eran únicos, no encontrándose ninguno multicéntrico.

El $90 \%$ es de naturaleza benigna y menor de $2 \mathrm{~cm}$, localizándose por igual en las 3 regiones del páncreas (1). En nuestra serie sólo existe 1 caso relacionado con el MEN-1 (8,4\%), no tenemos ninguna forma maligna, y el tamaño medio es de $2 \mathrm{~cm}$, localizándose por igual en cabeza y cuerpo-cola.

Todos nuestros pacientes presentaron el cuadro clínico clásico de hipoglucemia con síntomas neuroglucopénicos que cedían tras la ingesta de glucosa.

El gran caballo de batalla en el tratamiento de este tipo de tumores consiste en el diagnóstico topográfico preoperatorio. A pesar del gran número de pruebas de imagen de que disponemos, el pequeño tamaño de los insulinomas, hace que el diagnóstico topográfico no sea siempre posible. En una serie de estudios se llega a la conclusión de que la ecografía, el TAC y la RMN presentan una sensibilidad en el diagnóstico preoperatorio de este tipo de tumores que ronda el 50-60\% (2-11).

En nuestra casuística, la TAC se ha mostrado efectiva en la localización de la neoplasia en más de un $80 \%$ de las ocasiones, debido en gran medida a la mejor calidad de las imágenes obtenidas con el TAC helicoidal multicorte.

Los insulinomas expresan receptores para somatostatina en menos del 50\% de los casos, de ahí que la gammagrafía con octreótido no será una prueba útil en muchos pacientes (2). En nuestra serie se realizó a 6 pacientes, y esta sólo fue positiva en 2 de ellos. Mejores resultados se obtienen con la realización de la arteriografía mesentérica (positiva en los 2 casos) y con la ecoendoscopia (positiva en los 3 casos en que se realizó).

Estamos de acuerdo con la mayor parte de los autores en que esta prueba (ecoendoscopia) debería ser indispensable como prueba de diagnóstico preoperatorio en todos los pacientes, alcanzando una sensibilidad del $80-90 \%$ en diversas series (2-12).

Quizá la arteriografía sería menos indispensable por su carácter invasivo y probablemente sólo debería utilizarse en casos seleccionados de difícil diagnóstico preoperatorio.

A pesar de que la TAC se ha demostrado menos efectiva y con una tasa de aciertos variables en el diagnóstico preoperatorio de este tipo de tumores, en nuestra experiencia ha sido de gran ayuda, y creemos que es una prue- ba que se debería realizar en todos los casos, no sólo para intentar localizar la lesión, sino también para descartar la presencia de metástasis a distancia.

En cualquier caso, muchos de los artículos revisados $(2,4,5,7,8,11)$ consideran que la prueba diagnóstica más sensible en el diagnóstico topográfico de este tipo de tumores es la exploración y palpación del páncreas durante la cirugía, junto con la realización de una ecografía intraoperatoria, rondando tasas del $100 \%$ en el diagnóstico, que hemos confirmado en nuestra experiencia. Hoy en día creemos que esta prueba es de obligatoria realización para efectuar una cirugía de calidad de este tipo de tumores.

La cirugía constituye el tratamiento de elección, siendo la enucleación el procedimiento más frecuentemente realizado (13). Las tasas de curación oscilan entre un 7598\% tras la cirugía, en función de su naturaleza (benignamaligna) y de la extirpación completa del tumor (2). En nuestra serie en 9 casos se realizó una enucleación y en 3 casos una pancreatectomía distal. La técnica dependió de la localización tumoral, prefiriéndose la enucleación en los casos de localización cefálica, lesión córporo-caudal pequeña y distante del conducto pancreático principal; y la resección en tumores distales, de mayor tamaño o muy próximos al conducto pancreático principal.

En todos los casos se efectuó un abordaje convencional a través de una incisión subcostal bilateral, y consideramos de enorme ayuda la utilización del bisturí harmónico.

La realización de una resección pancreática a ciegas en caso de no localizar el tumor durante la cirugía, se debe abandonar, terminando la cirugía en ese momento, y derivando al paciente a un centro de referencia en este tipo de cirugía $(2,14)$.

Desde la introducción de la laparoscopia en el tratamiento del insulinoma en 1996 por Gagner (15) y Sussman (16), son cada vez más frecuentes los artículos publicados en la literatura de este tipo de abordaje, demostrando que esta técnica es sobre todo eficaz en los tumores pequeños, solitarios y de localización distal (1723). La mayor dificultad radica en el diagnóstico de localización intraoperatoria, que constituye la causa más frecuente de conversión (hasta un 30\% en grandes series), sin embargo la utilización de la ecografía laparoscópica está contribuyendo a disminuir estos problemas. A pesar del mayor auge de la cirugía laparoscópica, no existe un consenso claro en la literatura acerca de sus indicaciones. Nosotros no tenemos experiencia en este tipo de abordaje quirúrgico en el momento actual.

En cuanto a las complicaciones postoperatorias, no presentamos ningún caso de mortalidad y únicamente se produjeron 2 casos de fístula pancreática y 1 caso de pseudoquiste pancreático, que se resolvieron de manera conservadora, por lo que presentamos una tasa de morbilidad (25\%) comparable a la de otras series (13).

Durante el seguimiento efectuado a partir del año 2000 en que se inicia el estudio, no presentamos ningún caso 
de recidiva tras 48 meses, desapareciendo los síntomas hipoglucémicos y normalizándose las cifras de glucemia en todos los pacientes.

Las recurrencias tras la cirugía son poco frecuentes, apareciendo fundamentalmente en los casos asociados al MEN-1 (2).

\section{CONCLUSIÓN}

En nuestra experiencia, creemos aconsejable la realización de TAC abdominal y ecoendoscopia como pruebas de imagen previas a la cirugía.

Sin embargo, la técnica de elección sería la palpación del páncreas y la realización de una ecografía intraoperatoria.

La cirugía es curativa en el $100 \%$ de los casos, y la técnica efectuada dependerá del tamaño, localización y distancia del tumor al conducto pancreático, así como su relación con el síndrome MEN-1.

\section{BIBLIOGRAFÍA}

1. Sitges-Serra A, Fernández-Cruz L. Tumores endocrinos del páncreas. Manual de la Asociación Española de Cirujanos. Ed. Panamericana; 2004. p. 688-95.

2. Tucker ON, Crotty PL, Conlon KC. The management of insulinoma. Br J Surg 2006; 93: 264-75.

3. Apodaca-Torrez FR, Treviño T, Lobo EJ, Goldenberg A, Benvenuto MR. Insulinoma de páncreas. Cir Esp 2006; 80: 3-8.

4. Cougat P, Goudet P, Peix JL, Henry JF, Sarfati E, Proye C, et al. Insulinomas in multiple endocrine neoplasia type 1. Report of a series of 44 cases by the multiple endocrine neoplasia study group. Ann Chir 2000; 125: 118-23.

5. Mabrut JY, Lifante JC, Cherki S, Sin S, Berger N, Peix JL. Is preoperative localitation of insulinomas necessary? Ann Chir 2001; 126: 850-6.

6. Pitre J, Soubrane O, Dousset B, Palazzo L, Chapuis Y. Pancreatic echo-endoscopy and preoperative localitation of insulinoma. Ann Chir 1998; 52: 369-73

7. Chirletti P, Caronna R, Tamburrano G, Mellozzi M, Bonifacino A,
Catalano C, et al. Topographic diagnosis and surgical treatment of insulinoma. Chir Ital 2000; 52: 11-6.

8. Fendrich V, Bartsch DK, Langer P, Zielke A, Rothmund M. Diagnosis and surgical treatment of insulinoma experience in 40 cases. Dtsch Med Wochenschr 2004; 129: 941-6.

9. Zimmer T, Scheruble H, Faiss S, Stolzel U, Riecken EO, Wiedenmann B. Endoscopic ultrasonography of neuroendocrine tumors. Digestion 2000: 62 (Supl. 1): 45.

10. Proye Ch, Malvaux P, Pattou F, Filoche B, Godchaux JM, Maunoury V. Noninvasive imaging of insulinomas and gastrinomas with endoscopic ultrasonography and somatostatin receptor scintigraphy. Surgery 1998; 124: 1134-4.

11. Service FJ, Mc Mahon MM, O'Brien PC, Ballard DJ. Functioning insulinoma-incidence, recurrence and long-term survival of patients: a 60 -year study. Mayo Clin Proc 1991; 66: 711.

12. Maluenda MD, Pou JM, Varas MJ, Turró J, Abad R, Espinos J. Ecoendoscopia: implicaciones clínicas después de un período de más de 16 años de existencia. Rev Esp Enferm Dig 1999; 91: 209-22.

13. Grants CS. Insulinoma. Surg Oncol Clin North Am 1998; 7: 819-44.

14. Hirshberg B, Libutti SK, Alexander HR, Bartlett DL, Cochran C, Livi A, et al. Blind distal pancreatectomy for occult insulinoma, an inadvisable procedure. J Am Coll Surg 2002; 194: 761-4.

15. Gagner M, Pomp A, Herrera MF. Early experience with laparoscopic resections of islet cell tumors. Surgery 1996; 120: 1051-4.

16. Sussman LA, Christie R, Whittle DE. Laparoscopic excision of distal pancreas including insulinoma. Aust N Z L Surg 1996; 66: 414-6.

17. Fernández-Cruz L, Astudillo E, Martínez I, César-Borges G, Orduña D, Halperin I, et al. Cirugía laparoscópica en pacientes con insulinoma esporádico e insulinomas asociados a neoplasia endocrina múltiple tipo 1. Cir Esp 2004; 75: 189-95.

18. Berrospi F, Ruiz E, Chavez I, Celis J .Tratamiento laparoscópico del insulinoma pancreático. Técnica quirúrgica y resultados perioperatorios. Rev Gastroenterol Perú 2005; 25: 366-70.

19. Samaniego C, Martínez P, Sánchez R. Manejo del insulinoma. Reporte de una serie de casos. Gastr Latinoam 2005; 16: 250-4

20. Iihara M, Kanbe M, Okamoto T, Ito Y, Obara T. Laparoscopic ultrasonography for resection of insulinomas. Surgery 2001; 130: 108691.

21. Ayav A, Bresler L, Brunaud L, Boissel P, SFCL (Societe Francaise de Chirurgie Laparoscopique), AFCE (Association Francophone de Chirurgie Endocrinienne). Laparoscopic approach for solitary insulinoma: a multicentre study. Langenbecks Arch Surg 2005; 390: 13440.

22. Toniato A, Meduri F, Foletto M, Avogaro A, Pelizzo M. Laparoscopic treatment of benign insulinomas in the body and tail of the pancreas: a single-center experience. World J Surg 2006; 30: 1916-9.

23. Lo CY, Chan WF, Lo CM, Fan ST, Tam PK. Surgical treatment of pancreatic insulinomas in the era laparoscopy. Surg Endosc 2004; 18: 297-302. 